\title{
Wide Binaries in TGAS: Search Method and First Results
}

\author{
Jeff J. Andrews ${ }^{1,2}$, Julio Chanamé ${ }^{3,4}$ and Marcel A. Agüeros ${ }^{5}$ \\ ${ }^{1}$ Foundation for Research and Technology - Hellas, IESL, Voutes, 71110 Heraklion, Greece \\ email: andrews@physics.uoc.gr \\ ${ }^{2}$ Physics Department \& Institute of Theoretical \& Computational Physics, \\ University of Crete, 71003 Heraklion, Crete, Greece \\ ${ }^{3}$ Instituto de Astrofísica, Pontificia Universidad Católica de Chile, \\ Av. Vicuña Mackenna 4860, 782-0436 Macul, Santiago, Chile \\ ${ }^{4}$ Millennium Institute of Astrophysics, Santiago, Chile \\ ${ }^{5}$ Department of Astronomy, Columbia University, \\ 550 West 120th Street, New York, NY 10027, USA
}

\begin{abstract}
Half of all stars reside in binary systems, many of which have orbital separations in excess of 1000 AU. Such binaries are typically identified in astrometric catalogs by matching the proper motions vectors of close stellar pairs. We present a fully Bayesian method that properly takes into account positions, proper motions, parallaxes, and their correlated uncertainties to identify widely separated stellar binaries. After applying our method to the $>2 \times 10^{6}$ stars in the Tycho-Gaia astrometric solution from Gaia DR1, we identify over 6000 candidate wide binaries. For those pairs with separations less than 40,000 AU, we determine the contamination rate to be $\approx 5 \%$. This sample has an orbital separation $(a)$ distribution that is roughly flat in log space for separations less than $\sim 5000 \mathrm{AU}$ and follows a power law of $a^{-1.6}$ at larger separations.
\end{abstract}

Keywords. binaries: visual, astrometry

\section{Introduction}

Binary systems exist with orbital separations extending from $\sim 10 R_{\odot}$ to $\sim$ pc. Unresolved binaries at the smallest separations are typically found by identifying radial velocity variations indicative of orbital motion, while binaries at intermediate separations can be identified by observing the stars' motions through their orbits over many years (e.g., Raghavan et al. 2010). At the largest separations, pairs of nearby stars are typically identified as being in a binary by matching their proper motions; these common proper motion pairs have separations from $10^{2}$ to $\geqslant 10^{4}$ AU (Luyten 1971).

Beyond $\approx 10^{3} \mathrm{AU}$, the components of wide binaries are not expected to interact significantly over their lifetimes. These pairs can be considered mini-open clusters (Binney \& Tremaine 1987) and are uniquely powerful for answering questions that cannot be addressed by other stellar systems. For example, wide binaries consisting of white dwarfs, subdwarfs, or M-dwarfs with FGK main sequence companions have been used to calibrate metallicity scales and age-rotation relations (e.g., Lépine et al. 2007). Other white dwarf-main sequence pairs and double white dwarfs have been used to determine the initial-to-final mass relation for white dwarfs (e.g., Zhao et al. 2012). At the widest separations, the orbits of stellar binaries are dynamically affected by interactions with passing stars, giant molecular clouds, and the Galactic tide, each of which acts to stochastically disrupt them (Weinberg et al. 1987). These binaries have been used as probes of the gravitational potential of their host, including the contribution of dark objects and of dark matter in general (e.g., Yoo et al. 2004). 


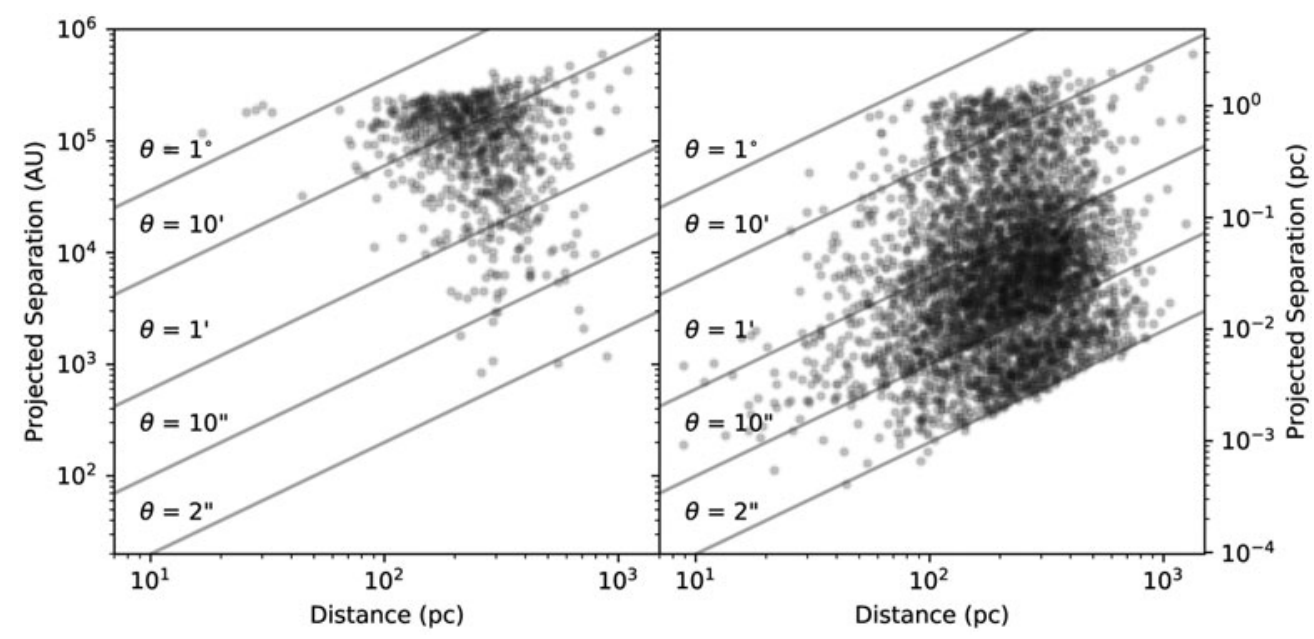

Figure 1. The distance and projected separation distribution of pairs from our random alignment catalog (left panel) and our catalog of $>6000$ candidate pairs (right panel). The locus of points at projected separations $\sim 1 \mathrm{pc}$ are primarily random alignments since they exist in both panels. At separations less than $4 \times 10^{4} \mathrm{AU}$, there are few random alignments. Comparison between the two panels indicates that the contamination rate of random alignments in our sample of wide binaries is $\approx 5 \%$ for pairs with separations less than $4 \times 10^{4}$ AU.

Due to its extremely precise astrometry, the final Gaia catalog will produce accurate positions, proper motions, and parallaxes for more than a billion stars in the Galaxy. This data set offers a unique opportunity to robustly identify a sample of wide stellar binaries. Using the Tycho-Gaia Astrometric Solution (TGAS) from the first Gaia data release (Lindegren et al. 2016), we define a Bayesian statistical method that accounts for the correlated uncertainties in Gaia's astrometry to identify wide binaries.

\section{Method Overview}

We identify stellar pairs using the five dimensions of phase space provided for each star in the TGAS catalog: right ascension $(\alpha)$, declination $(\delta)$, proper motion in right ascension and declination $\left(\mu_{\alpha}, \mu_{\delta}\right)$ and parallax $(\varpi)$.

We simplify the problem by reparameterizing the 10 observables (five for each star) into eight for each pair; instead of having a separate sky position and proper motion for each star, we use one position and proper motion for the stellar pair and include an angular separation $(\theta)$ and a proper motion difference $(\Delta \mu)$ between the two stars. We then split the observables into one set containing the $(\alpha, \delta)$ and $\left(\mu_{\alpha}, \mu_{\delta}\right)$ position of the stellar pair, and another set containing the difference between the observed values for each of these parameters as well as the individual parallaxes $\left(\theta, \Delta \mu, \varpi_{1}\right.$, and $\left.\varpi_{2}\right)$.

Our method relies on a Bayesian formalism with two classes: every pair of stars is either a random alignment of unassociated stars or a genuine wide binary. For each stellar pair, we calculate two prior probabilities and two likelihoods. Since the number of stellar binaries in a sample scales with the size of the sample, $N$, while the number of randomly aligned pairs scales with $N(N-1) / 2$, any arbitrary pair has a strong prior of being a random alignment. The exact value of this prior, which depends on the local density of a pair's position and proper motion, needs to be determined for every pair.

The probability of random alignments scales with the phase space volume of stars multiplied by the local stellar density in that phase space. Therefore, the random alignment 

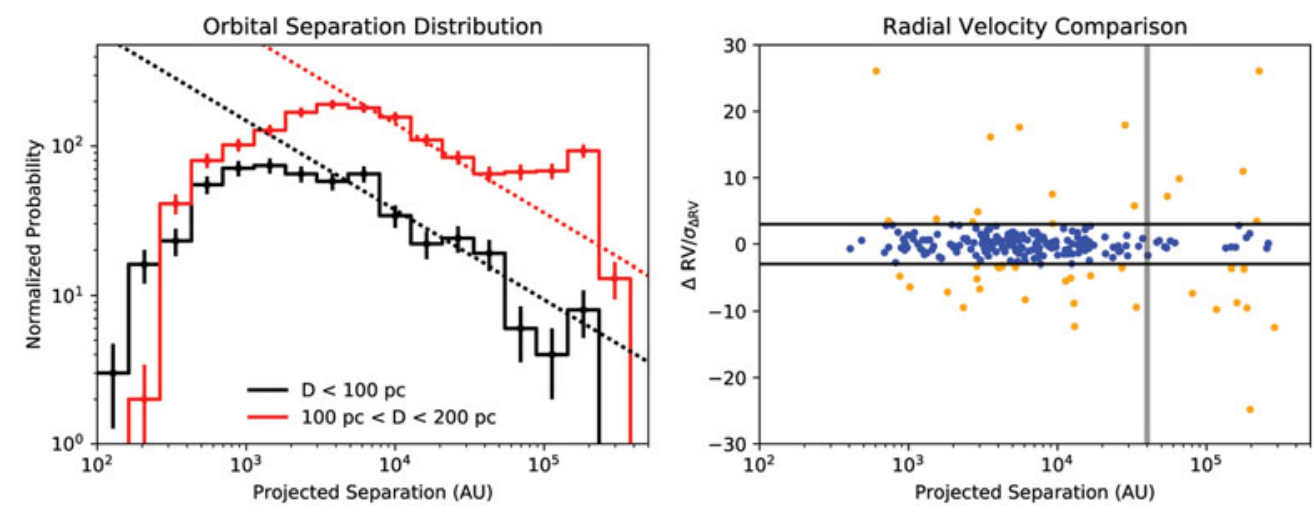

Figure 2. Left panel - The orbital separation distribution of our candidate pairs at distances less than $100 \mathrm{pc}$ (black) and at distances between 100 and $200 \mathrm{pc}$ (red). At large separations, the distribution scales with $a^{-1.6}$ (dotted lines), while at smaller separations, the distribution flattens and scales with $a^{-1}$. Right panel - Comparison between the radial velocities of the two stars in the subset of our candidate pairs observed by the RAVE survey. Most pairs have radial velocities consistent at the $3 \sigma$ level (blue), bounded by the gray horizontal lines. Several pairs have somewhat discrepant radial velocities (yellow). Particularly for those pairs with projected separations less than $4 \times 10^{4} \mathrm{AU}$, these pairs could indicate the presence of unresolved binaries in our sample; some of our wide binaries may in fact be hierarchical triple systems.

likelihood increases linearly with $\theta$ and $\Delta \mu$, and scales with the local density in $(\alpha, \delta)$ and $\left(\mu_{\alpha}, \mu_{\delta}\right)$ space. Calculating the corresponding likelihood that a particular stellar pair is a genuine binary has traditionally been determined as the likelihood that the two stars have matching proper motions. TGAS astrometry is precise enough that we need to account for the fact that orbital motion may cause slight differences in the proper motions of the two stars. For details of how we account for these differences as well as the exact forms of our two prior probabilities and likelihoods see Andrews et al. (2017).

Random alignment catalog. To estimate the rate of contamination in our sample, we adapt the procedure of Lépine \& Bongiorno (2007) and generate a catalog of randomly aligned wide binaries by shifting each star by $+2^{\circ}$ in declination and +3 mas $\mathrm{yr}^{-1}$ in both $\mu_{\alpha}$ and $\mu_{\delta}$. We then apply our algorithm to identify matches between this shifted star and the original, unshifted catalog. Every resulting pair is a random alignment.

Our wide binary sample. Figure 1 shows the distance and projected separation of candidate pairs in the two catalogs of matched binaries produced using our Bayesian method. The left panel shows those pairs produced due to random alignments while the right panel shows our catalog of genuine binaries. The locus of points in both panels at projected separations of $\sim 1 \mathrm{pc}$ indicates that our sample is dominated by random alignments at these separations. At separations less than $\approx 4 \times 10^{4}$ AU, our sample is dominated by genuine pairs. By comparing the numbers of pairs with $\theta<4 \times 10^{4} \mathrm{AU}$ in both panels, we estimate the rate of contamination due to random alignments to be $\approx 5 \%$.

\section{First Results}

Orbital separation distribution. The left panel of Figure 2 shows the orbital separation distribution for pairs within the nearest $100 \mathrm{pc}$ (black) and for pairs at distances between 100 and 200 pc (red). These samples are subject to strong observational biases, but using a more sophisticated analysis in which we account for selection effects, we determine two things: first, the distribution of the sample at relatively larger separations is well characterized by a $P(a) \sim a^{-1.6}$ power law (indicated by the dotted lines). The increase 
in the sample of binaries at distances between 100 and $200 \mathrm{pc}$ at $s \sim 10^{5} \mathrm{AU}$ is due to contamination from random alignments. Were this uptick genuine, a corresponding increase would be observed in the sample at distances within 100 pc. Second, the turnover at $s \sim 3 \times 10^{3} \mathrm{AU}$ is a genuine characteristic of the sample; at smaller separations, the distribution follows one closer to Öpik's law, i.e., $P(a) \sim a^{-1}$. These results are in general agreement with those observed in the wide binary samples of Chanamé et al. (2004) and Lépine \& Bongiorno (2007).

Radial velocities. Large scale radial velocity surveys such as the Radial Velocity Experiment (RAVE; Kunder et al. 2017) provide a useful test of our method; the radial velocities of the components of genuine wide binaries should be consistent. The right panel of Figure 2 shows the radial velocity difference, scaled by the uncertainty in this difference, between the two stars in wide binaries for those pairs in which RAVE radial velocities are available. Horizontal lines bound the region in which the two radial velocities are consistent at the $3 \sigma$ level. Pairs at projected separations smaller than $4 \times 10^{4}$ $\mathrm{AU}$ (gray vertical line) have a contamination rate of $\approx 5 \%$, and indeed, the stars overwhelmingly have consistent radial velocities. There are a number of pairs (yellow) which have inconsistent radial velocities despite their low contamination fraction. Some of the components of these wide binaries may contain unresolved binaries, and may therefore be hierarchical triple (or higher order) systems.

\section{Future Expectations}

The second data release from Gaia will contain $>10^{9}$ stars with five astrometrically measured dimensions of phase space. Identifying associated stellar pairs in such a large catalog presents a unique data analysis challenge. Nevertheless, our method is designed for scalability and use with a high performance computing cluster. Using the increase in catalog size as a guide, this data release may contain between $10^{5}$ and $10^{6}$ wide binaries.

\section{Acknowledgements}

This work has made use of data from the European Space Agency (ESA) mission Gaia (https://www. cosmos.esa.int/gaia), processed by the Gaia Data Processing and Analysis Consortium (DPAC, https://www.cosmos.esa.int/web/gaia/dpac/ consortium). Funding for the DPAC has been provided by national institutions, in particular the institutions participating in the Gaia Multilateral Agreement.

\section{References}

Andrews, J. J., Chanamé, J., \& Agüeros, M. A. 2017, ArXiv preprint:1704.07829

Binney, J. \& Tremaine, S., 1987, Galactic Dynamics

Chanamé, J. \& Gould, A., 2004, ApJ, 601, 289

Kunder, A., et al. 2017, AJ, 153, 75

Lépine, S. \& Bongiorno, B., 2007, AJ, 133, 889

Lépine, S., Rich, R. M., \& Shara, M. M., 2007, ApJ, 669, 1235

Lindegren, L., et al., 2016, A\&A, 595, A4

Luyten, W. J., 1971, ApESSS, 11, 49

Raghavan, D., et al., 2010, ApJS, 190, 1

Weinberg, M. D., Shapiro, S. L., \& Wasserman, I., 1987, ApJ, 312, 367

Yoo, J., Chanamé, J., \& Gould, A., 2004, ApJ, 601, 311

Zhao, J. K., Oswalt, T. D., Willson, L. A., Wang, Q., \& Zhao, G., 2012, ApJ, 746, 144 